\title{
PEDESTRIAN LATERAL ACTION ON LIVELY FOOTBRIDGES: A NEW LOAD MODEL
}

\author{
F. Venuti, PhD student, L. Bruno, Associate Prof., P. Napoli, Full Prof., Department of Structural Engineering and \\ Geotechnics, Politecnico di Torino, Torino, Italy.
}

\section{Summary}

This paper proposes a new load model to predict the lateral force exerted by pedestrians walking on lively footbridges. The aim of the model is to take into account some important features of the synchronous lateral excitation phenomenon, which so far has not been fully understood or modelled, e.g. the distinction between synchronization among pedestrians, due to crowd density, and between the pedestrians and the structure, caused by deck oscillations; the triggering of the lock-in phenomenon and its self-limited nature. The proposed load model has been tested with reference to two crowd events that were recorded on the T-bridge in Japan (1993) and on the Millennium Bridge in London (2001). The results obtained with the present model are compared to results predicted by other load models found in literature and are then discussed.

\section{Introduction}

The phenomenon of synchronous lateral excitation caused by pedestrians walking on footbridges has attracted increasing public attention in the last few decades from the earliest cases in the nineties [1] to the well-known case of the London Millennium Bridge [2]. Up to date, this kind of dynamic lateral load has never led to structural failure, but has often caused discomfort for the users and the temporary closure of the footbridges in order to provide proper countermeasures [2]-[3]. This reduced serviceability represents a severe problem for its economic and social outcomes. These reasons have recently motivated intense research activity, reviewed in [4], which represents the scientific background of design guidelines (e.g. [5]-[6]).

The problem of determining the force exerted by synchronized pedestrians is generally tackled using an empirical approach. Laboratory tests involving a single pedestrian walking on a moving platform (e.g. [7]) allow important data to be obtained on the lateral force exerted by one pedestrian and to estimate the mean probability that individuals will synchronise their step to the platform motion [2]. Moreover, the observation of videos recorded during crowd events on actual bridges [1] permits a qualitative estimate to be made of the synchronization phenomena that occur between people walking in a crowd.

Several models have been proposed using the aforementioned data to predict the lateral force exerted by pedestrians. Most of the models found in literature (e.g. the ones reviewed in [4]) are deterministic time-domain models, based on the assumption that both feet produce exactly the same periodic force. The load models proposed in the design codes also belong to this category. For example, three different load models are proposed in [5] to estimate the force exerted by a single pedestrian, by a group of pedestrians or by a crowd uniformly distributed along the footbridge deck, respectively.

To the authors' knowledge, many of the force models proposed have so far experienced some difficulties in taking into account some not negligible aspects of the problem, that is:

- the dependence of the pedestrian force on the two-way interaction between two systems, the crowd and the structure, which can be described by two variables, the pedestrian density and the footbridge lateral vibration. In this paper the deck velocity is retained as the vibration variable which mostly affects the pedestrian force, as indicated by some authors (e.g. [2]);

- the possibility of a inhomogeneous distribution of the crowd along the deck due to bottlenecks, congested traffic or other non-linear traffic phenomena;

- the existence of two kinds of synchronization, as recently pointed out in [8] and [9], one between the pedestrians and the structure and the other among the pedestrians. The latter takes place when the relative movement of the pedestrians is constrained because of high crowd density;

- the presence of different frequency components in the overall force;

- triggering of the lock-in phenomena and the resulting selflimited oscillations.

The aim of the present work is to propose a force model that gives due weight to the aforementioned features of the phenomenon. The proposed mathematical model assumes that crowd density and deck velocity are given, for instance by means of in situ measurements, computational simulations with a crowd-structure interaction model [10] or a priori chosen within a worst-case scenario approach. On one hand, the model is conceived to 
provide an accurate description of the phenomena and, on the other, to become a useful tool for footbridge designers and engineers, in order to predict footbridge behaviour under pedestrian loads.

The model was conceived on the basis of a macroscopic description of crowd dynamics, which means that the pedestrians are not viewed as single individuals but as clusters characterized by a mean walking velocity and a mean step frequency. Nevertheless, an adaptation of the model to a microscopic or statistical description of the crowd is possible. First, a detailed description of the model is given; then, its properties are evaluated and, finally, two applications are presented and discussed.

\section{Formulation of the model}

The proposed force model can be ascribed to the category of timedomain models. It is based on the assumption that the force exerted by a number $n$ of pedestrians walking along a portion of the bridge span is given by the sum of three components:

$$
F=F_{p s}+F_{p p}+F_{s}
$$

where $F_{p s}$ is the term due to the synchronization between the pedestrians and the structure, $F_{p p}$ is due to the synchronization among pedestrians and $F_{s}$ is the part due to uncorrelated pedestrians.

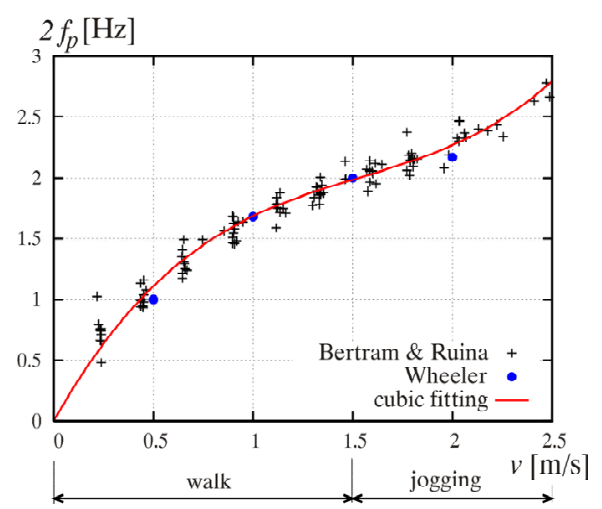

Figure 1: Relationship between step frequency and pedestrian velocity

$F_{p s}$ has the same frequency $f_{s}$ as the excited lateral structural mode, while the other two terms have the same frequency $f_{p}$ as the lateral pedestrian footstep. $f_{p}$ is assumed to vary as a function of the walking velocity $v$ (Figure 1), as reported by Bertram \& Ruina [11] and Wheeler [12].

In turn, the walking velocity $v$ can be expressed as a function of the crowd density $u$, and of the deck velocity $\zeta$ ( intended as the envelope of the velocity time history), as proposed in [10].
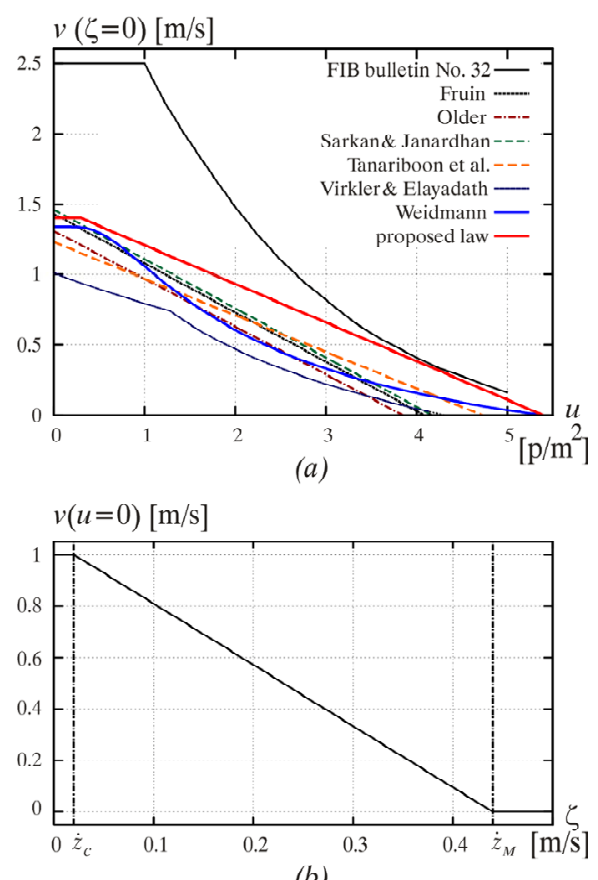

Figure 2: Relation between $v$ and $u(a)$ and $v$ and $\zeta(b)$

Several relations between $v$ and $u$, the so-called fundamental diagrams, have been proposed by different authors (e.g. [5], [13]) on the basis of experimental tests and the observation of real events (Figure 2a). It is interesting to notice that the only diagram reported in a design guideline [5] is clearly different from the other ones: it is based on only a few measurements referring to fast pedestrian transit in train stations, and can therefore be considered meaningless for the applications discussed in this paper. The slight differences that can be observed among the other diagrams are due to different measurement situations (location, travel purpose, pedestrian attributes). The linear fundamental diagram proposed by the authors has been conceived to describe the behaviour of pedestrians crossing the footbridge on the occasion of particular events and in the case of unidirectional flow.
As for the relation between $v$ and $\zeta$, a qualitative law is proposed (Figure $2 \mathrm{~b}$ ): pedestrian walk is unconstrained when $\zeta$ is less than a critical value $\dot{z}_{c}=0,02 \mathrm{~m} / \mathrm{s}$, inferred from the experimental data reported in [2]; the walking velocity linearly decreases as $\zeta$ grows and the pedestrians are no longer able to walk as $\zeta$ reaches a maximum admissible value $\dot{z}_{M}=$ $0,44 \mathrm{~m} / \mathrm{s}$ (suggested by Nakamura in [14]).

As a consequence of the aforementioned relations, the lateral step frequency $f_{p}$ is a function of both the crowd density $u$ and the lateral footbridge velocity $\zeta$ (Figure $3)$. This relation assures that, in the presence of excessive lateral deck motion or crowd density, pedestrians stop walking, so that the amplitudes of the terms $F_{p p}$ and $F_{s}$ are selflimited.

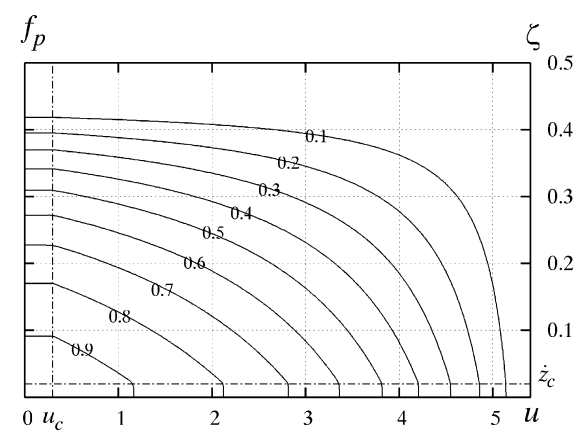

Figure 3: $f_{p}$ versus $u$ and $\zeta$

Each term of the overall force is weighted on the basis of phenomenological considerations, by means of three weights, $n_{p s}, n_{p p}$ and $n_{s}$, that can be considered, respectively, as the number of pedestrians in the cluster that are synchronized with the structure, synchronized to each other and uncorrelated:

$$
\begin{aligned}
& n_{p s}=n S_{p s} \\
& n_{p p}=n S_{p p}\left(1-S_{p s}\right) \\
& n_{s}=n-n_{p s}-n_{p p}
\end{aligned}
$$

where $S_{p s}$ and $S_{p p}$ are the synchronization coefficients, which both vary in the [l $\left.\begin{array}{ll}0 & 1\end{array}\right]$ range. Thanks to the distinction of pedestrians in three categories, the model is able to represent the triggering of lock-in: even though no one is synchronized 
with the structure, the presence of a high crowd density results in a lateral force that triggers the lateral vibration of the bridge.

$S_{p s}$ represents the degree of coupling between the crowd and the structure. This is a function of two variables: the structure lateral velocity $\zeta$ and the ratio $f_{r}=f_{p} / f_{s}$, where $f_{r}$ is defined in the [ll $\left.\begin{array}{ll}0 & 2\end{array}\right]$ domain: the lower bound depends on the minimum value of $f_{p}\left(f_{p}=0\right)$, when the walking velocity is null; the upper bound was obtained from the laboratory tests reported in [7], according to which pedestrians are not influenced by structural oscillations under $0,6 \mathrm{~Hz}$ and the maximum recorded lateral walking frequency is $1,1 \mathrm{~Hz}$.

The variation of $S_{p s}$ versus $\zeta$ (Figure 4a) is given by a fitting of the Dallard et al. experimental data [2], by means of the interpolating function:

$$
S_{p s}(\zeta)=1-e^{-b\left(\zeta-\dot{z}_{c}\right)}
$$

Pedestrians start to synchronize with the structure for values of $\zeta$ higher than $\dot{z}_{c}$, and they are completely synchronized when $\zeta$ reaches the maximum value $\dot{z}_{M}$.
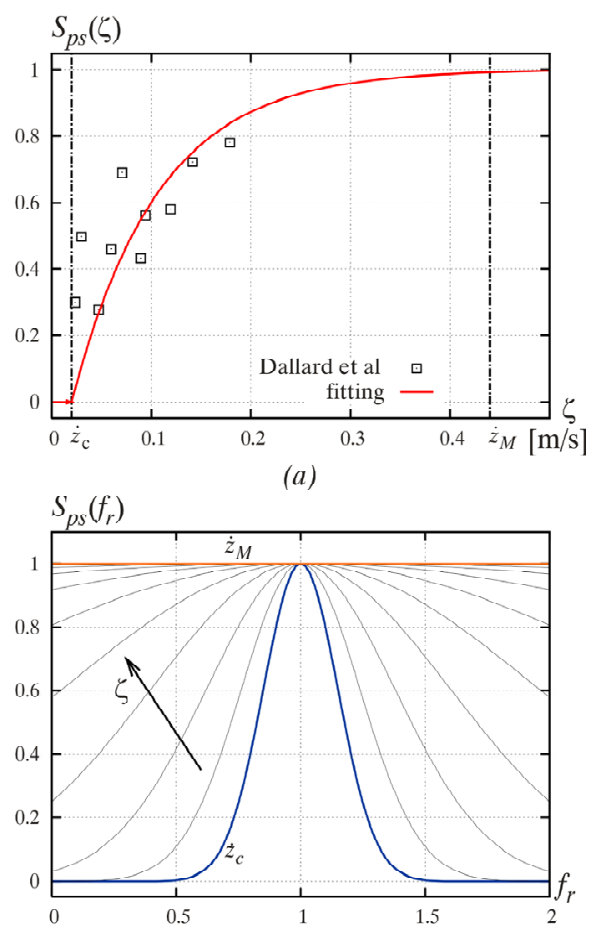

(b)

Figure 4: Sps versus $\zeta(a)$ and $f_{r}(b)$
$S_{p s}\left(f_{r}\right)$ is supposed to have a normal distribution, with a variance that grows when $\zeta$ increases. This means that, for increasing values of $\zeta$, the pedestrians who walk with a step frequency that is different from $f_{s}$ gradually become involved in the synchronization phenomenon. For $\zeta$ $=\dot{z}_{M}$, everyone is synchronized with the structure, whatever the value of $f_{r}$ (Figure $4 \mathrm{~b}) . S_{p s}\left(f_{r}\right)$ is defined as:

$$
\begin{aligned}
& S_{p s}\left(f_{r}\right)=e^{\left[-\gamma\left(f_{r}-1\right)^{2}\right]} \\
& \gamma(\zeta)=50 e^{(-40 \zeta)}
\end{aligned}
$$

The synchronization coefficient $S_{p s}\left(\zeta, f_{r}\right)$ is given by the product of equations (3) and (4).

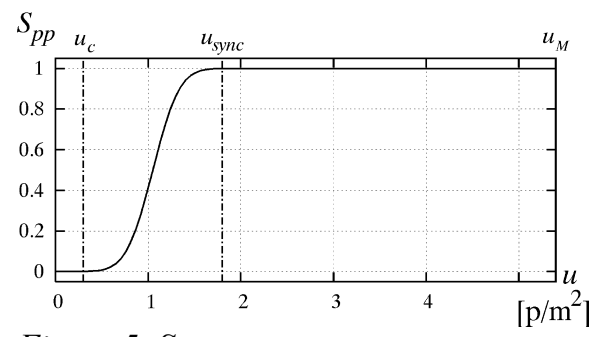

Figure 5: Spp versus u

The coefficient $S_{p p}$ (Figure 5) represents the degree of synchronization among pedestrians and, because of the lack of experimental data, it has been defined in a qualitative way as a function of the crowd density $u$ $\left[\mathrm{ped} / \mathrm{m}^{2}\right]$ :

$S_{p p}(u)=\frac{1}{2}\left\{1+\operatorname{erf}\left[a u_{M}\left(u-u^{*}\right)\right]\right\}$,

$u^{*}=\frac{\left(u_{s y n c}-u_{c}\right)}{2}$

where:

- $a=3,14$;

- $u_{c}$ is a critical density that represents the upper limit for unconstrained free walking. According to [5], its value is set to $0,3 \mathrm{ped} / \mathrm{m}^{2}$

- $u_{M}$ is the maximum admissible density, corresponding to a pedestrian velocity equal to zero. Different values of $u_{M}$ are proposed in literature (e.g. [13]): in the following $u_{M}=5,4 \mathrm{ped} / \mathrm{m}^{2}$;

- $\quad u_{\text {sync }}$ is the value that corresponds to the total synchronization of pedestrians. Its value is estimated to be $1,8 \mathrm{ped} / \mathrm{m}^{2}$, according to the maximum density recorded on the Tbridge [3].

It is worthwhile pointing out that the proposed laws for the synchronization coefficients $S_{p s}$ and $S_{p p}$ can be interpreted as cumulative density functions of an exponential and a Gaussian probability density function, respectively. From this point of view, the model can be adapted to a statistical description of the phenomenon as soon as a greater amount of data is available.

The first component of the total force, $F_{p s}$, can be written, according to [7], as the sum of a component in phase with the structure acceleration $\ddot{z}$ and another one in phase with the velocity $\dot{z}$ :

$$
F_{p s}=n_{p s}\left[\bar{F}_{\ddot{z}} \sin \left(2 \pi f_{s} t\right)+\bar{F}_{\dot{z}} \sin \left(2 \pi f_{s} t\right)\right]
$$

The amplitudes of the two components (Figure 6) are defined by means of piecewise functions.
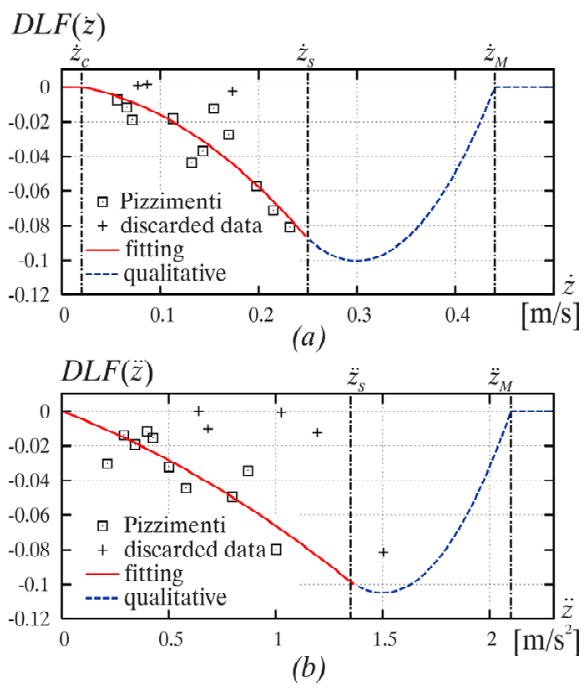

Figure 6: DLFs of the components in phase with velocity (a) and acceleration (b)

The first branch comes from a quadratic fitting of the experimental data concerning the medium Dynamic Load Factors (DLFs) of the in-phase and out-of-phase components [7]. The data corresponding to DLFs close to zero for non null $\dot{z}$ or $\ddot{z}$, and to $\dot{z}$ or $\ddot{z}$ above their serviceability limit (that is, $\dot{z}_{s}=0,25 \mathrm{~m} / \mathrm{s}$ and $\ddot{z}_{s}=1,35 \mathrm{~m} / \mathrm{s}^{2}$ [14]) have been discarded. The second branch is defined 
qualitatively, based on the following assumptions:

- the DLFs reach their maximum when the velocity and the acceleration exceed their serviceability limit;

- the DLFs decrease to zero when the velocity and the acceleration reaches maximum values, above which pedestrians stop walking, i.e. $\dot{z}_{M}=0,44 \mathrm{~m} / \mathrm{s}$ and $\ddot{z}_{M}=2,1 \mathrm{~m} / \mathrm{s}^{2}$ [14]. This trend also guarantees that the amplitude of $F_{p s}$ is self-limited as is the overall structural response.

The second force component, $F_{p p}$, is defined as:

$$
F_{p p}=n_{p p} \bar{F}_{s} \sin \left(2 \pi f_{p} t\right)
$$

where $\bar{F}_{s}$ is the medium amplitude of the force exerted by a single pedestrian in the case of a motionless deck, whose DLF=0,04 [4].

Finally, the component $F_{s}$ is determined according to the model proposed by Matsumoto et al. [15], who found that the force due to $n$ pedestrians walking with Gaussian distributed frequencies and random phases is $\sqrt{n}$ higher than the force due to a single pedestrian. Therefore, $F_{s}$ becomes:

$F_{s}=\sqrt{n_{s}} \bar{F}_{s} \sin \left(2 \pi f_{p} t\right)$

\section{Properties of the model}

The properties of the proposed force model have been evaluated by means of a sensitivity study on the two main variables, $\zeta$ in the $\left[\begin{array}{ll}0 & 0,5\end{array}\right] \mathrm{m} / \mathrm{s}$ interval and $u$ in the $\left[\begin{array}{ll}0 & u_{M}\end{array}\right]$ ped $/ \mathrm{m} 2$ range. The simulations are performed assuming $f_{s}=0,93 \mathrm{~Hz}$ (the first lateral natural frequency of the T-bridge in Japan [16]) and a platform velocity time history with constant amplitude $\zeta$ :

$\dot{z}=\zeta \cos \left(2 \pi f_{s}\right)$

This type of simulation can be compared to an experimental test on a moving treadmill, except that the single pedestrian is substituted by a cluster of pedestrians. As an example, Figure 7 graphs the time histories of the three force components and the overall force in the case of $u=1,3 \mathrm{ped} / \mathrm{m}^{2}$ and $\zeta=0,06$ $\mathrm{m} / \mathrm{s}$ (conditions recorded on the $\mathrm{T}$ bridge [16]).
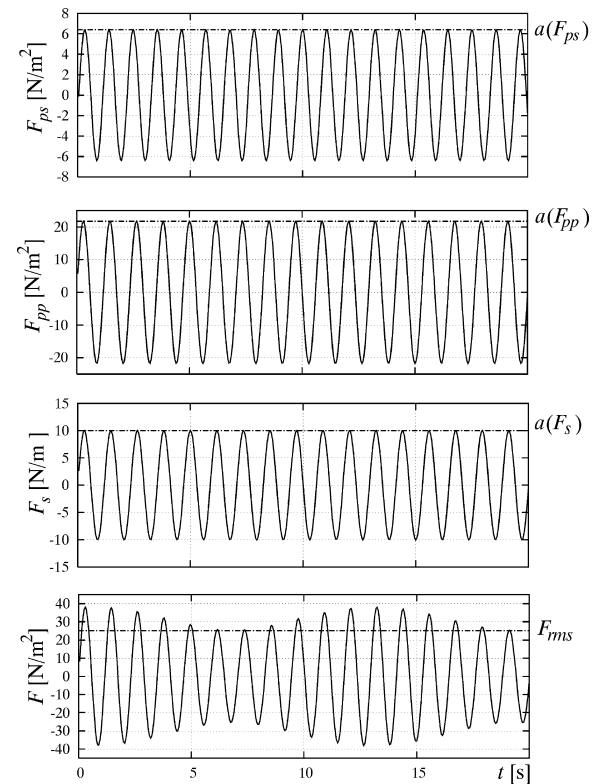

Figure 7: Time histories of the force components
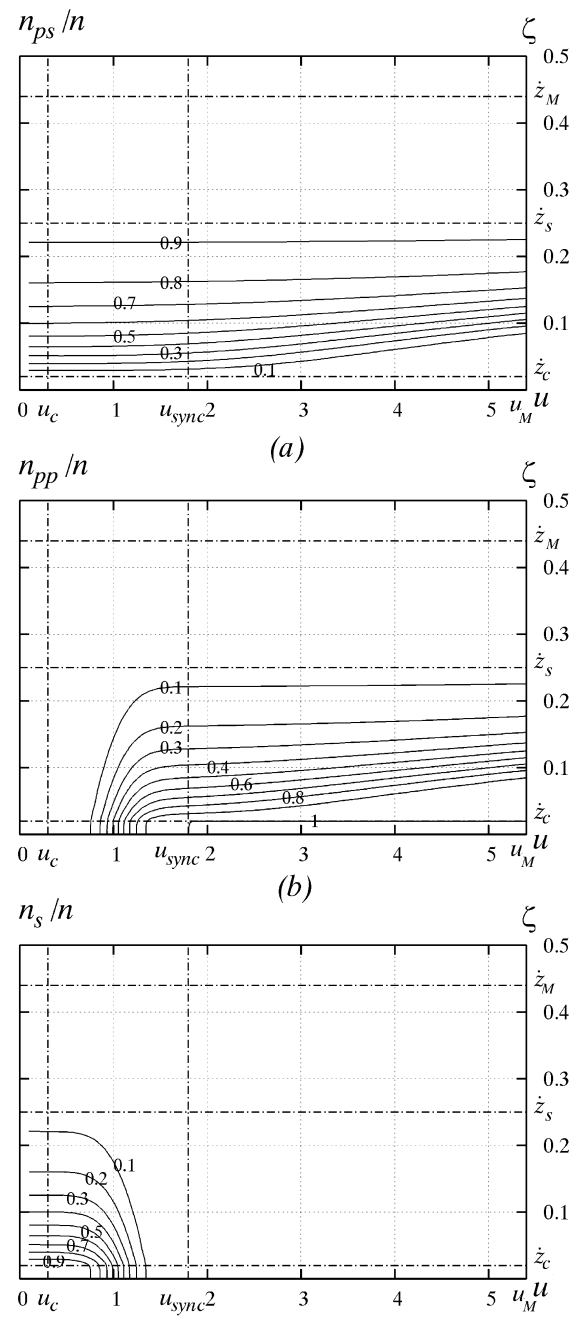

(c)

Figure 8: Weights of the force components scaled with respect to $n$

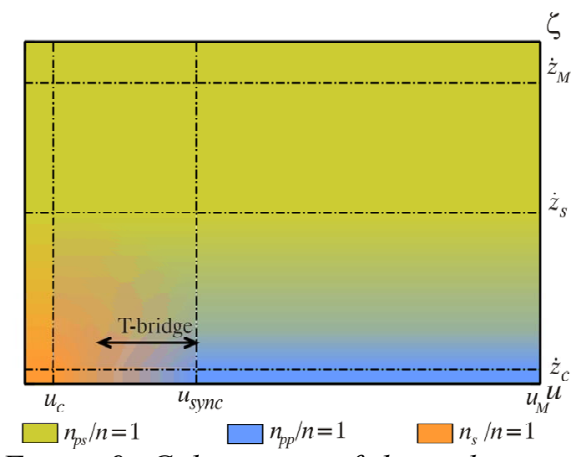

Figure 9: Colour map of the pedestrian synchronization
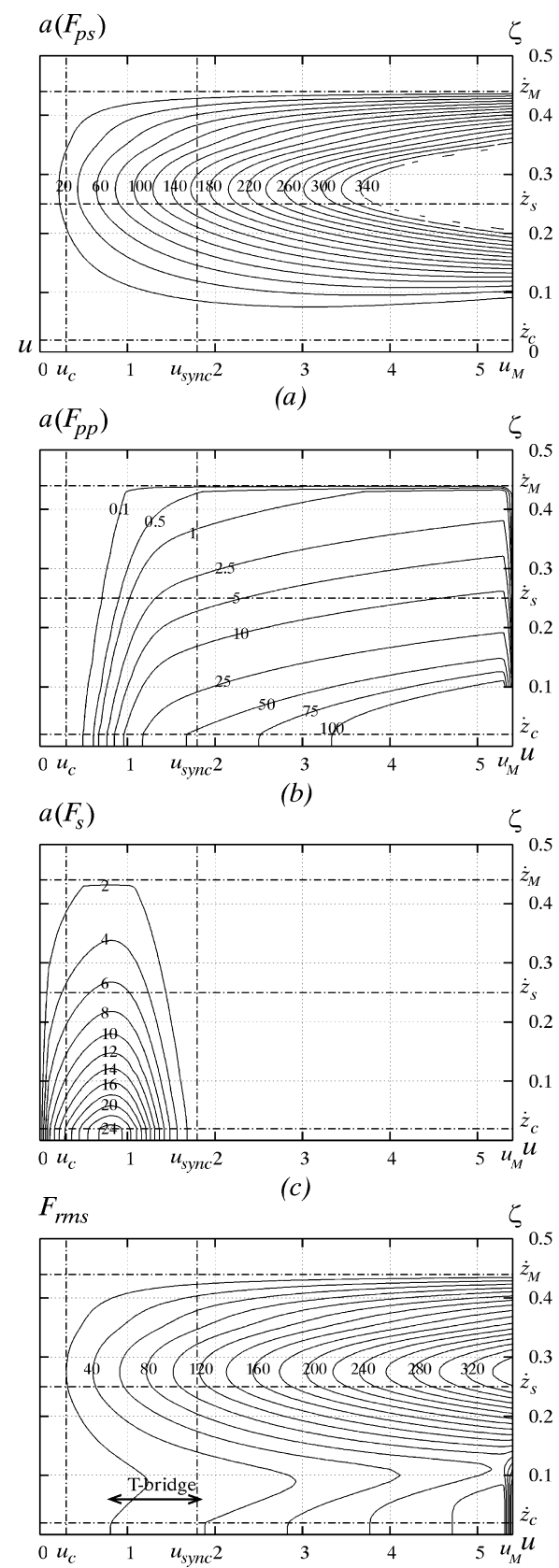

(d)

Figure 10: Amplitudes of the force components and rms value of the overall force

Figure 8 shows the weights of the force components, scaled with respect to the number of pedestrians $n$, versus $\zeta$ and $u$. The following considerations can be made: 
- $\quad n_{p s}$ is much more sensitive to the deck velocity $\zeta$, than to the crowd density $u$ and grows monotonically as $\zeta$ increases and as $u$ decreases; it reaches the highest value for $\zeta>\dot{z}_{s}$, whatever the value of $u$ (Figure $8 \mathrm{a}$ );

- $\quad n_{p p}$ has the opposite trend to $n_{p s}$ : it grows as $u$ increases and as $\zeta$ decreases. For $u>u_{s y n c}\left(S_{p p}=1\right), n_{p p}$ is complementary to $n_{p s}$ (Figure $8 \mathrm{~b}$ );

- $n_{s}$ is obtained from subtraction of the other two terms, therefore it reaches the maximum value when $\zeta$ and $u$ are under their critical values and is null for $\zeta>\dot{z}_{s}$ and $u>u_{\text {sync }}$, when all the pedestrians are synchronized (Figure 8c). A colour map of the three weights in the $u-\zeta$ plane (Figure 9) can be obtained for each structure, in order to quickly estimate, in the preliminary design phase, which kind of synchronization will play the main role.

Figure 10 represents the amplitudes $a$ of the three force terms (in $\mathrm{N} / \mathrm{m}^{2}$ ) and of the overall force $F$, which is expressed in terms of its root mean square $(\mathrm{rms})$ value $\left(F_{r m s}\right)$, versus $\zeta$ and $u$.

It can be observed that:

- the evolution of $F_{p s}$ versus $\zeta$ is influenced by both $n_{p s}$ and by the distribution of the DLFs (see Figure 6), which determines its non monotonic trend. The dependence of $F_{p s}$ on $u$ is almost linear, i.e. its amplitude grows linearly as the number of pedestrians increases (Figure 10a);

- the amplitude of $F_{p p}$ has the same evolution as $n_{p p}$, since it comes from the product of $n_{p p}$ and the constant $\bar{F}_{s}$. It is worthwhile pointing out that $F_{p p}$ goes abruptly to zero when $f_{p}=0$, that is, for $u=u_{M}$ or $\zeta$ $>\dot{z}_{M}$ : this means that the pedestrians who have stopped do not exert lateral excitation and the self-limited nature of the deck response is guaranteed (Figure 10b). A similar consideration can be drawn for $F_{s}$, which also has a non monotonic evolution versus $u$ (Figure 10c);

- the evolution of $F_{r m s}$ versus $u$ and $\zeta$ is not trivial, since it comes from the sum of periodic signals with different frequencies and it therefore cannot be determined by simply summing the three component amplitudes (Figure 10d). The amplitudes of the force components can also be analysed with respect to their frequency content (Figure 11). The term $F_{p s}$ evolves in time with a constant frequency equal to $f_{s}$; the component $F_{p p}$, instead, has a frequency $f_{p}$ which depends on both $u$ and $\zeta$ (see Figure 3 ). When the two variables are under their critical values, $f_{p}$ is at its maximum value, while, as $u$ and $\zeta$ grow, the walking velocity decreases and $f_{p}$ decreases in turn.
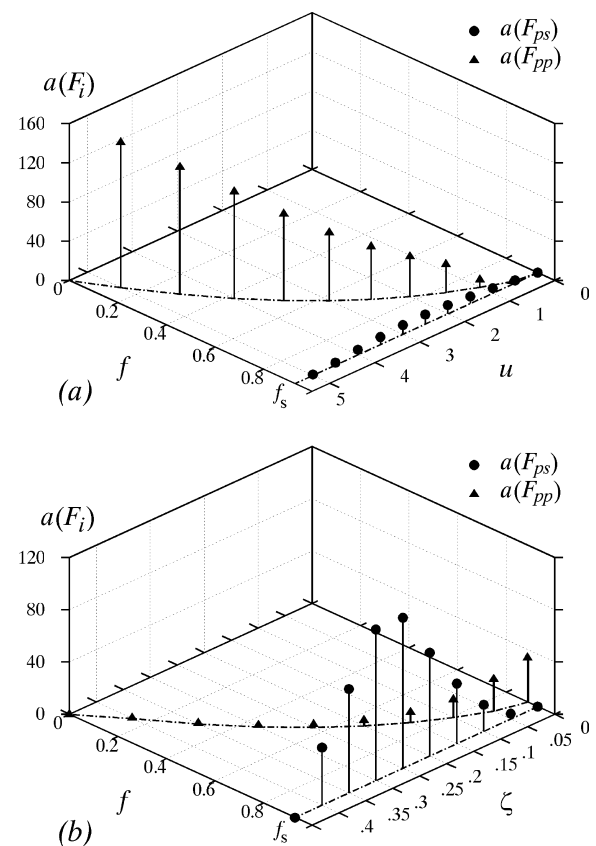

Figure 11: Amplitudes of $F_{p s}$ and $F_{p p}$ versus $f_{p}$, for $\zeta=0,06 \mathrm{~m} / \mathrm{s}$ (a) and $u=1,3$ $\mathrm{ped} / \mathrm{m}^{2}$ (b)

\section{Applications}

- The force model has been tested adopting the conditions recorded in two crowd events on the T-bridge in Japan (Figure 12) and on the Millennium Bridge in London (Figure 13).

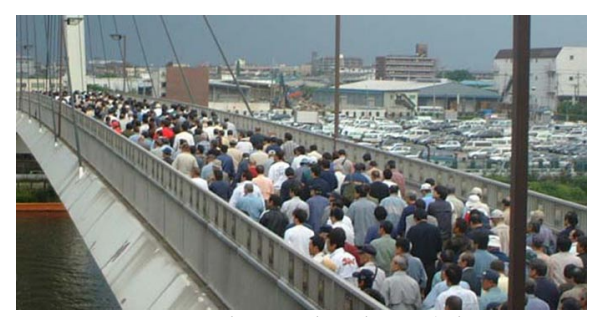

Figure 12: The T-bridge (photo was taken by S. Nakamura [3])
The data used for the simulations are the following:

- T-bridge: $u=0,8 \div 1,8 \mathrm{ped} / \mathrm{m}^{2} ; f_{s}$ $=0,93 \mathrm{~Hz} ; \zeta=0,06 \mathrm{~m} / \mathrm{s}$ [1]-[16];

Millennium bridge: $u=1,3 \div 1,5$ $\mathrm{ped} / \mathrm{m}^{2} ; f_{s}=0,8 \mathrm{~Hz}$ (south span); $\zeta=0,25 \mathrm{~m} / \mathrm{s}$ [2].

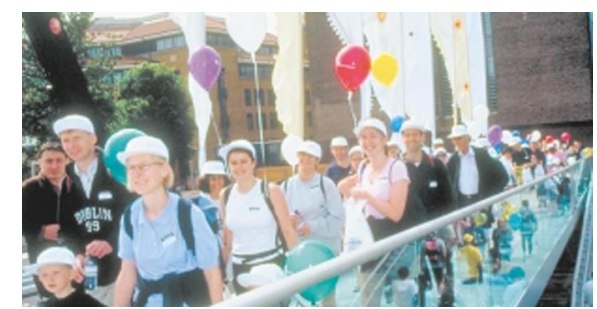

Figure 13: The Millennium Bridge (from [2])

The single force per pedestrian [ $\mathrm{N} /$ ped] obtained with the model is compared to that predicted with the force models proposed in [1]-[16][2] (Table 1): the ranges of the reported values refer, respectively, to the lower and higher crowd densities used for the simulations. It should be pointed out that, since the cited models do not distinguish between the two types of synchronization, the predicted force is considered to be only due to the pedestrian-structure synchronization and it is therefore compared to the term $F_{p s}$.

Table 1: Comparison between the proposed force model and those found in literature (forces in N/ped)

\begin{tabular}{clc}
\hline & \multicolumn{1}{c}{ Model } & $\boldsymbol{a}\left(\boldsymbol{F}_{\boldsymbol{p s}}\right)$ \\
\hline T-br. & Fujino et al [1] & 7 \\
& Nakamura [16] & 4,7 \\
& Dallard et al [2] & 18 \\
& Proposed & $5,7 \div 4,7$ \\
\hline M. br. & Dallard et al [2] & 75 \\
& Proposed & 81 \\
\hline
\end{tabular}

First, it is should be noticed that the results provided by the models in literature are rather different for the T-bridge: this is probably due to the fact that each model is based on the data recorded on a particular footbridge and it can not therefore be extended to all other structures. For instance, Nakamura \& Kawasaki observed that the model they proposed for the T-bridge only agreed with that of Dallard et al. for deck lateral velocities under 0,015 $\mathrm{m} / \mathrm{s}$. The proposed model, instead, has a more general validity, since it 
is not based on one single event but on the phenomenological description of the components of the coupled system in their fundamental constitutive laws.

The amplitudes of the term $F_{p s}$ predicted by the proposed model are very similar to those estimated by Nakamura \& Kawasaki for the Tbridge and by Dallard et al. for the Millennium bridge, respectively: this fact highlights the wide applicability of the model.

Table 2 provides a deeper insight into the other results obtained with the model. The correspondence between the simulated results and the actual data can clearly be seen looking at the force frequency content. In the case of the T-bridge, the estimated step lateral frequency $f_{p}(0,89 \div 0,8 \mathrm{~Hz})$ is very close to the deck lateral frequency $(0,93 \mathrm{~Hz})$, therefore the two components $F_{p p}$ and $F_{s}$ also excite the first lateral mode. As for the Millennium bridge, since $f_{p}$ is close to $0,5 \mathrm{~Hz}$, it can be stated that the model is also able to predict what occurred on the central span, which vibrated at both the first $(0,5 \mathrm{~Hz})$ and the second $(1 \mathrm{~Hz})$ lateral modes. Hence, it can be argued that the central span second mode $(1 \mathrm{~Hz})$ was excited by the crowd-structure interaction, while the first was excited by the synchronization of pedestrians and by uncorrelated pedestrians.

Finally, let us consider the number of synchronized pedestrians. Even though both events are characterized by high crowd density, as the two structures have different slenderness, the Millennium bridge shows higher oscillations than the T-bridge, which means a greater number of pedestrians synchronized with the structure. Since only a few pedestrians are captured in the structure-induced synchronization on the T-bridge, the crowd density plays a leading role in determining the overall force (see also Figure 9).

\section{Conclusion}

The proposed force model satisfies the preset objectives. The main features of the pedestrian lateral excitation phenomenon are taken into account, in particular the fact that both the crowd density and the motion of the footbridge deck influence pedestrian behaviour. The model is also accurate compared to the data and models found in literature, and can therefore be considered as a general predictive tool for the design and analysis of footbridges under pedestrian loads. Because of its versatility, it can be used for different purposes and with different degrees of accuracy: during the preliminary design phase, it allows the worst load scenario to be outlined if the expected values of crowd density and deck velocity are a priori chosen; in the final design phase, it can be used to determine the load that should be applied to the structural model or to a complete computational simulation of the crowd-structure interaction [10]; once the footbridge has been built, the model is able to define the pedestrian force on the basis of the deck velocity and crowd density measured in situ during actual events.

It should be pointed out that some of the laws presented in this paper come from qualitative considerations and are not adequately supported by experimental data. For this reason, the model, which constitutes a valid reference framework, can certainly be improved with a proper tuning of the parameters by means of ad hoc conceived experimental tests. Finally, the model framework is suitable to be extended to pedestrian vertical loading.

\section{Acknowledgement}

The authors wish to express their acknowledgement to professors $\mathrm{F}$. Ricciardelli, Y. Fujino and S. Nakamura for kindly making their experimental data available.

\section{References}

[1] FUJINO Y., PACHECO B. M., NAKAMURA S., WARNITCHAI P. Synchronization of human walking observed during lateral vibration of a congested pedestrian bridge. Earth. Eng. Struct. Dyn., No. 22, pp. 741-758, 1993.

[2] DALLARD P., FITZPATRICK T., LE BOURVA S., LOW A., RIDSDILL R. M., WILLFORD M. The London Millennium
Footbridge. The Structural Engineer, No. 79(22), pp. 17-33, 2001.

[3] NAKAMURA S., FUJINO Y. Lateral vibration on a pedestrian cable-stayed bridge, Structural Engineering International, No. 12(4), pp.295-300, 2002.

[4] ŽIVANOVICH S., PAVIC A., REYNOLDS P. Vibration serviceability of footbridges under human-induced excitation: a literature review. J. of sound and vibration, No. 279, pp. 1-74, 2005.

[5] Federation International du Beton. Guidelines for the design of footbridges, FIB Bulletin No. 32, Lausanne, 2006.

[6] SETRA/AFGC. Passerelles piétonnes Evaluation du comportement vibratoire sous l'action de piétons. Guide méthodologique. Paris, 2006.

[7] PIZZIMENTI A.D., Experimental analysis of the lateral pedestrian-induced mechanism of excitation of footbridges, $\mathrm{PhD}$ Thesis, Università degli studi di Catania, 2004 (in italian).

[8] RICCIARDELLI F. Lateral loading of footbridges by walkers. Proceedings Footbridge 2005, Venice, 2005.

[9] VENUTI F., BRUNO L., BELLOMO N. Crowd structure interaction: dynamics modelling and computational simulations. Proceedings Footbridge 2005, Venice, 2005.

[10] VENUTI F., BRUNO L., BELLOMO $\mathrm{N}$. Crowd dynamics on a moving platform: mathematical modelling and application to lively footbridges. Mathematical and Computer Modelling, No. 45, pp. 252-267, 2007

[11] BERTRAM J. E., RUINA A. Multiple walking speed-frequency relations are predicted by constrained optimisation. $J$. theor. Biol., No. 209, pp. 445-453, 2001.

[12] WHEELER J. Prediction and control of pedestrian induced vibration in footbridges. ASCE J. of the Structural Division, No. 108, pp. 2045-2065, 1982.

[13] DAAMEN W. Modelling passenger flows in public transport facilities. $\mathrm{PhD}$ Thesis, Delft University of technology, 2004.

[14] NAKAMURA S. Field measurement of lateral vibration on a pedestrian suspension bridge. The Structural Engineer, No. 81(22), pp. 22-26, 2003.

[15] MATSUMOTO Y., NISHIOKA T-, SHIOJIRI H., MATSUZAKI K. Dynamic design of footbridges. IABSE Proceedings, No. P-17(78), pp. 1-15, 1978.

[16] NAKAMURA S., KAWASAKI T. Lateral vibration of footbridges by synchronous walking, $J$. of Constructional Steel Research, No. 62, pp. 1148-1160, 2006. 
Table 2: Results obtained with the proposed model (forces in N/ped)

\begin{tabular}{cccccccc}
\hline Benchmark & $\boldsymbol{a}\left(\boldsymbol{F}_{\boldsymbol{p s}}\right)$ & $\boldsymbol{a}\left(\boldsymbol{F}_{\boldsymbol{p p}}\right)$ & $\boldsymbol{a}\left(\boldsymbol{F}_{s}\right)$ & $\boldsymbol{f}_{\boldsymbol{p}}$ & $\boldsymbol{F}_{\boldsymbol{r} \boldsymbol{s} \boldsymbol{s}}$ & $\boldsymbol{n}_{\boldsymbol{p s}} / \boldsymbol{n}$ & $\boldsymbol{n}_{p p} / \boldsymbol{n}$ \\
\hline T-bridge & $5,7 \div 4,7$ & $2,5 \div 19,9$ & $24,9 \div 0$ & $0,89 \div 0,8$ & $19,7 \div 14,5$ & $0,33 \div 0,38$ & $0,08 \div 0,66$ \\
\hline M. bridge & 81 & $1,9 \div 2,1$ & $2,6 \div 1$ & $0,56 \div 0,54$ & $57,7 \div 57,6$ & 0,93 & $0,06 \div 0,07$ \\
\hline
\end{tabular}

